\title{
DIFICULDADES VIVENCIADAS POR MÃES DE RECÉM-NASCIDOS PREMATUROS DURANTE A PERMANENCIA PROLONGADA EM AMBIENTE HOSPITALAR
}

\section{DIFFICULTIES LIVED BY MOTHERS OF PREMATURE NEWBORN BABIES DURING PROLONGED STAY IN HOSPITAL}

\author{
DIFICULTADES VIVENCIADAS POR MADRES DE RECIÉN-NASCIDOS \\ PREMATUROS DURANTE PERMANENCIA PROLONGADA EM AMBIENTE \\ HOSPITALARIO \\ Divanice Contim ${ }^{1}$, Cristina Ranuzi ${ }^{2}$, Jurema Ribeiro Luiz Gonçalves ${ }^{3}$, Carolina Feliciana \\ Bracarense $^{4}$, Jesislei Bonolo do Amaral $^{5}$, Nara dos Santos Costa ${ }^{6}$
}

\begin{abstract}
RESUMO
Objetivo: identificar as dificuldades vivenciadas pelas mães de recém-nascidos prematuros durante a permanência prolongada no ambiente hospitalar. Método: estudo descritivo, de caráter qualitativo, realizado em um Hospital Federal Universitário. Participaram nove mães de recém-nascidos prematuros. Os dados foram coletados por meio de entrevistas semiestruturadas. O material foi submetido à técnica de análise do Discurso do Sujeito Coletivo. Resultados: dos relatos construiu-se três discursos, sendo: adaptação ao hospital; sentimentos que envolvem a internação prolongada e; preocupação com a família. Conclusão: as dificuldades encontradas pelas mães são: falta de experiência, insegurança, cansaço, estresse, ansiedade, saudade de casa, preocupação e a adaptação a rotina hospitalar.
\end{abstract}

Palavras-chave: Recém-Nascido; Hospitalização; Mães.

\begin{abstract}
Objective: to identify the difficulties lived deeply for the just-born mothers of premature during the permanence drawn out in the hospital environment. Method: descriptive study, of qualitative character, carried through in a University Federal Hospital. Nine just-born mothers of premature had participated. The data had been collected by way of interviews semistructuralized. E the material submitted to the technique of analysis of the Speech of the Collective Citizen. Results: of the stories one constructed three speeches, being: Adaptation to the hospital; Feelings that involve drawn out internment e; Concern with the family. Conclusion: the difficulties found for the mothers are the lack of experience, unreliability, fatigue, estresse, anxiety, homesickness of house, concern and the adaptation the hospital routine.
\end{abstract}

Keywords: Infant, Newborn; Hospitalization; Mothers.

\footnotetext{
1 Doutora em Ciências pela Universidade Federal de São Paulo- Docente do Curso de Graduação em Enfermagem da Universidade Federal do Triângulo Mineiro (UFTM). E-mail: d.contim@uol.com.br

2 Enfermeira pela Universidade Federal do Triangulo Mineiro (UFTM). E-mail: cristinaranuzi@gmail.com

3 Doutora em Enfermagem Psiquiátrica pela Escola de Enfermagem de Ribeirão Preto (EERP/USP) Docente do Curso de Graduação em Enfermagem e da Pós-Graduação Stricto sensu em Atenção à Saúde da Universidade Federal do Triângulo Mineiro (UFTM). E-mail: juremaluiz@hotmail.com.br

${ }^{4}$ Enfermeira. Mestre em Atenção à Saúde pela Universidade Federal do Triangulo Mineiro (UFTM). E-mail: carolinafbracarense@gmail.com

${ }^{5}$ Mestre em Enfermagem Fundamental pela Escola de Enfermagem de Ribeirão Preto - Universidade de São Paulo (EERP - USP). Docente do Curso de Graduação em Enfermagem da Universidade Federal do Triângulo Mineiro (UFTM). E-mail: jesisleimjlo@gmail.com

${ }^{6}$ Enfermeira. Mestre em Atenção à Saúde pela Universidade Federal do Triângulo Mineiro (UFTM). E-mail: narasc29@gmail.com
} 


\section{RESUMÉN}

Objetivo: identificar las dificultades vivenciadas por madres de recién-nascidos prematuros durante la permanencia prolongada en el ambiente hospitalario. Método: estudio descriptivo, de carácter cualitativo, realizado en un Hospital Federal Universitario. Participaron nueve madres de recién-nascidos prematuros. Los datos fueron colectados por medio de entrevistas semiestructuradas. El material fue sometido a la técnica de analice del Discurso del Sujeto Colectivo. Resultados: de los relatos se construyó tres discursos, siendo: Adaptación al hospital; Sentimientos que envuelven la hospitalización prolongada y; Preocupación con la familia. Conclusión: las dificultades encontradas por las madres son la falta de experiencia, inseguridad, cansancio, estrese, ansiedad, nostalgia de casa, preocupación y la adaptación a la rutina hospitalaria.

Descriptors: Recién-nascido; Hospitalización; Madres.

oito a 36 dias, segundo estudos realizados

\section{INTRODUÇÃO}

A Organização Mundial de Saúde pondera que ocorrem cerca de 15 milhões de nascimentos prematuros no mundo, número este que corresponde a mais de $10 \%$ do total de nascimentos. O Brasil ocupa a $10^{\mathrm{a}}$ posição no ranking dos países com maior número de nascimentos prematuros. ${ }^{1}$ Em 2011, 11,8\% dos bebês nascidos nesse país foram prematuros, trata-se de uma taxa extremamente alta, se comparada com outros países em mesma situação social e econômica. $^{2}$

Recém-nascidos prematuros possuem características específicas, como a termorregulação ineficaz, pele fina, em alguns casos precisam de nutrição enteral, parenteral, suporte ventilatório, punções venosas e monitorização. ${ }^{3}$ Em virtude dessa fragilidade e necessidade de cuidados intensivos geralmente demandam internação hospitalar prolongada.

O tempo de internação hospitalar nos casos de prematuridade pode variar de em diferentes regiões do país. ${ }^{4-6}$ A hospitalização prolongada faz a família vivenciar sentimentos controversos como: medo, insegurança e tristeza por não poder levar o filho para casa e proporcionar os primeiros cuidados; e de alegria, felicidade e conforto por saber que seu filho está recebendo uma assistência integral e com os recursos essenciais. ${ }^{7-8}$

Os sentimentos que emergem com a hospitalização do recém-nascido podem afetar significativamente os laços afetivos entre os pais e os filhos, por isso minimizar os sentimentos angustiantes e potencializar os de esperança é primordial para a recuperação do prematuro. Estratégias que visem valorizar a presença materna na unidade hospitalar precisam ser desenvolvidas e estimuladas. As mães acompanhantes devem ser incluídas nos cuidados cotidianos, o toque afetivo encorajado e a comunicação entre a equipe de saúde e a família instituída. ${ }^{8-9}$

Os profissionais de saúde, em 
especial os enfermeiros, precisam desempenhar ações que possibilitem e favoreçam a adequação progressiva da mãe e da família à realidade do nascimento e hospitalização de um filho prematuro. Há a necessidade de uma nova abordagem no atendimento neonatal, na qual deverão ser contemplados não apenas os aspectos das necessidades fisiológicas do prematuro, mas sobretudo aqueles ligados ao atendimento dos fatores emocionais que envolvem os cuidadores nos momentos das fragilidades presentes nas situações de prematuridade.

Nessa abordagem, parte-se do pressuposto de que essas mães demandam cuidados especiais, e, portanto, torna-se imprescindível conhecer os aspectos psicológicos e sociais vivenciados por elas com a hospitalização do filho na tentativa de oferecer suporte para o desempenho do papel materno nas unidades de internação saúde.

Mediante o exposto o presente estudo tem como objetivo identificar as dificuldades vivenciadas pelas mães de recém-nascidos prematuros durante a permanência prolongada no ambiente hospitalar.

\section{MÉTODO}

Trata-se de um estudo descritivo, de caráter qualitativo, realizado na unidade de internação pediátrica de um Hospital
Federal Universitário, em um município no interior de Minas Gerais.

Fizeram parte do presente estudo nove mães de recém-nascidos com mais de 14 dias de hospitalização. Essa amostra foi obtida por meio da saturação de dados, que consiste em um método contínuo de análise dos dados, que se inicia na fase de coleta de dados, a partir das questões abordadas pelo pesquisador. São realizadas pré-análises com o intuito identificar os tópicos abordados nas respostas dos entrevistados, quando novos tópicos não surgem nas respostas, obtém-se a saturação dos dados. ${ }^{10}$

Os dados foram obtidos por meio de entrevistas semiestruturada gravadas em formato de áudio. A entrevista contou com a seguinte interrogação "Como foi a experiência para a senhora vivenciar a internação prolongada do seu filho no ambiente hospitalar?". O período de coleta de dados foi de Fevereiro a Março de 2013.

Após a fase de coleta de dados o material resultante das entrevistas foi transcrito na íntegra. Procedeu-se a análise de acordo com a técnica do Discurso do Sujeito Coletivo (DSC) a qual trabalha com dados qualitativos de natureza verbal, obtidos por meio de fontes como entrevistas. $^{11}$

As expressões-chaves identificadas consistiram em trechos mais significativos do material coletado que expressavam a 
essência das respostas individuais e as ideias centrais que sintetizam o conteúdo discursivo manifestado. ${ }^{11} \mathrm{O}$ agrupamento das expressões-chave e ideias centrais semelhantes resultaram em três categorias, sendo: adaptação ao hospital; sentimentos que envolvem a internação prolongada e; preocupação com a família.

Os discursos-síntese serão apresentados na primeira pessoa do singular, nos quais o pensamento de um grupo ou coletividade aparece como um discurso individual. ${ }^{11}$ Os Discursos construídos serão apresentadas entre aspas ao longo do texto.

O projeto de pesquisa foi aprovado pelo Comitê de Ética e Pesquisa com Seres Humanos da Universidade Federal do Triângulo Mineiro, com o protocolo número 2127/2011; obedecendo os preceitos éticos da Resolução 466/2012.

\section{RESULTADOS}

A idade das nove mães que fizeram parte do estudo variou de 17 a 39 anos. Entre os recém-nascidos cinco eram do sexo feminino e quatro do sexo masculino, o peso ao nascer oscilou entre $895 \mathrm{Kg}$ e $2390 \mathrm{~kg}$, idade gestacional entre 29 e 35 semanas e o tempo de internação mínimo foi de 14 dias e o máximo de nove meses e dezesseis dias.

Apresenta-se o Discurso do Sujeito Coletivo 1: adaptação ao hospital. Neste discurso foram agrupadas cinco expressões-chave que fazem alusão ao processo adaptativo no cenário hospitalar. As entrevistadas relataram que o início da hospitalização foi assustador, porém tal situação foi atenuada pelo cotidiano da internação e a convivência com as demais mães.

No começo foi muito difícil, não tinha experiência com nada, nem com neném ainda, me sentia desesperada e insegura por ela ser muito pequena, mas aos poucos fui me adaptando a tudo e agora está sendo fácil. Conhecer outras mães como eu foi muito bom, nós nos unimos, conversamos muito e isso ajuda (E1, E2, E4, E5, E7).

O Discurso do Sujeito Coletivo 2: sentimentos que envolvem a internação prolongada. É composto por seis expressões-chave, abrange os sentimentos que permeia o processo de hospitalização duradoura.

A internação é um misto de tristeza e alegria, tristeza por minha filha estar aqui e alegria por estar viva. Foi uma surpresa, um susto, quando vi todos aqueles aparelhos fiquei com muito medo dela não sair da Unidade de Terapia Intensiva $e$ isso me preocupava muito, chorava demais. A internação acaba com a gente, é cansativo $e$ estressante ficar aqui todo esse tempo, a vida da gente para. Não esperava ficar tanto tempo, a ansiedade para poder ir embora e não precisar voltar mais é muito grande (E1, E3, E5, E6, E8, E9).

O Discurso do Sujeito Coletivo 3: 
preocupação com a família. Refere-se que além de conviver com as condições de saúde em que a criança se encontra e da necessidade de se adaptar ao ambiente hospitalar é preciso, também, enfrentar uma situação de desorganização familiar. Foi composta por oito expressões-chave.

A saudade de casa é muito grande, fico aqui preocupada com minha outra filha que está em casa. Ela pergunta muito por mim, mas como não posso ir vê-la, apenas conversamos por telefone (E1, E3, E4, E5, E6, E7, E8, E9).

\section{DISCUSSÃO}

A adaptação dessas mães ao hospital perpassa pelas relações de amizade que ali se estabelece com a troca de experiência, com o apoio nos momentos de desespero, angústia, medo, ansiedade e o com o consolo de dividirem problemas semelhantes. Nesse sentido as mães, constroem uma rede de solidariedade e amizade entre si, motivadas pelas necessidades e experiências em comum. Este cotidiano gera novas possibilidades de relacionamento e de vínculos, expressado no convívio diário, preocupações de umas com as outras, e compartilhando as alegrias e as tristezas.

A permanência materna no hospital durante a internação do filho prematuro carece de atenção, apoio, ajuda e de troca de experiências, sendo as outras mulheres ali presentes às únicas companhias para compartilhar as dores, os medos e os vários conflitos vivenciados. Essa reciprocidade reforça diariamente a esperança e consolida laços de amizade. ${ }^{12}$

A família sente-se mais confiante para vivenciar este momento de internação quando partilha experiências com os profissionais de saúde e com outras famílias. O estreitamento destes vínculos propícia a aquisição de informações sobre a condição de saúde do filho, além de envolver os pais no ato de cuidar. ${ }^{13}$

No discurso das mães foi expresso que a hospitalização do filho fez emergir sentimentos opostos, ao mesmo tempo em que desfrutavam da alegria da vida, também provavam da tristeza em ver o filho com aparelhos e em uma unidade de terapia intensiva. Além disso, o momento da internação foi descrito como cansativo, estressante e fonte de ansiedade e angústia.

Ao confiar à equipe de saúde os cuidados do seu filho, as mães vivenciam sentimentos de impotência e incapacidade. O longo do processo de hospitalização potencializa a angústia dos pais, devido à condição clínica do filho, e ao aparato tecnológico dispensado ao tratamento. Estes sentimentos são gerados frente à internação, diminuindo a autoestima materna e podem evoluir para depressão, fato que afetará a formação de um vínculo afetivo entre o binômio mãe-filho. ${ }^{9,14}$ 
Em um estudo que comparou dois grupos de mães de prematuros e de bebês a termo, revelando a ocorrência, em uma parcela proporcionalmente maior em comparação com outros estudos, de sintomas clinicamente significativos de ansiedade (75\%) e depressão (50\%) em mães de bebês prematuros. Já entre as mães de bebês a termo, $65 \%$ não apresentavam sintomas clinicamente significativos de ansiedade e tampouco depressão. Estes resultados corroboram a literatura, sugerindo que a prematuridade tende a ter impacto negativo na saúde mental da mulher que vivencia essa situação. ${ }^{15}$

A ansiedade vivenciada por essas mães parece estar ligada a um estado emocional transitório relacionado a acontecimentos recentes, como por exemplo, o nascimento e hospitalização do filho, marcados por sentimentos desagradáveis de tensão e apreensão em relação à internação do recém-nascido. ${ }^{14-}$ 15 Observa-se redução significativa do número de mães com ansiedade ou depressão após a alta hospitalar relacionada com a diminuição na preocupação quanto à sobrevida da criança. ${ }^{14}$ Estes estavam relacionados a acontecimentos recentes, como por exemplo, o nascimento e hospitalização do filho. ${ }^{15}$

Ainda que esses sintomas de ansiedade e depressão sejam transitórios, é preciso estar atento para as situações que intensifiquem tais sentimentos, visando minimizá-las para que esses sintomas não se tornem crônicos. Pois, a cronicidade de tal manifestação pode afetar negativamente a saúde mental das mães, prejudicando e dificultando os cuidados ao recémnascido.

As mães entrevistadas neste estudo salientaram que além dos sentimentos oriundos da internação prolongada precisam gerenciar a unidade familiar e se sentem preocupadas por estarem ausentes em suas outras funções.

A outra face da internação são as modificações no cotidiano, no orçamento financeiro e doméstico, a preocupação com a família, com os outros filhos, que se veem obrigados a conviver com este distanciamento inesperado e muitas vezes por tempo indeterminado. ${ }^{15}$ Essa rotina entre casa e hospital faz com que o recémnascido torne-se o principal foco do cuidado materno quando comparado aos demais filhos. ${ }^{16}$

$$
\text { A hospitalização prolongada }
$$
decorrente do nascimento prematuro desperta nos pais sentimentos negativos, de culpa, medo e importância, os quais favorecem o surgimento do estresse e ansiedade que podem comprometer a relação afetiva dos pais com o bebê. Esta situação desencadeia notáveis 
impactos na vida familiar e por esta razão necessita de uma atenção holística, a qual englobe além da mãe os demais membros da família. ${ }^{17-18}$

\section{CONSIDERAÇÕES FINAIS}

Neste estudo, as dificuldades
identificadas nos discursos maternos,
frente a permanência prolongada no
ambiente hospitalar foram: falta de experiência, insegurança, cansaço, estresse, sentimentos de tristeza, ansiedade, saudade de casa, preocupação, necessidade de adaptação a rotina hospitalar e a transferência da responsabilidade de cuidar da família para outro familiar. O convívio com as outras mães se mostrou de grande importância no processo de enfrentamento.

Compreender a experiência materna, planejar uma assistência de enfermagem que favoreça a qualidade de vida do filho e da mãe, visar o bem estar da família como condição importante para garantir o bem estar do paciente, permite uma ampla visão do cuidar frente à assistência em saúde.

Este estudo teve como principal limitação ter sido realizado em um único hospital que atua no cuidado ao recémnascido prematuro no interior de Minas Gerais o que não permite generalização dos dados.
Recomenda-se a promoção de grupos de mães, atividades de orientação ao cuidado do recém-nascido, levantamento de dúvidas, estimulo a troca de experiências, a união e o estreitamento dos vínculos de amizade estabelecidos nas unidades de internação neonatal e pediátrica.

\section{Colaborações}

Contim D e Ranuzi C contribuíram para concepção do trabalho, análise, interpretação dos dados, redação do artigo e aprovação final da versão a ser publicada. Gonçalves JRL, Bracarense CF, Amaral JB e Costa NS contribuíram para concepção do trabalho, redação do artigo e aprovação final da versão a ser publicada.

\section{REFERÊNCIAS}

1. World Health Organization. Born too soon: the global action report on preterm birth. Geneva: World Health Organization; 2012.

2. Unicef Brasil. Consultoria: pesquisa para estimar a prevalência de nascimentos pré-termo no Brasil e explorar possíveis causas. Unicef Brasil; 2013.

3. Ministério da Saúde (BR). Atenção à saúde do recém nascido. Guia para os profissionais da saúde. Brasília (DF):

Ministério da Saúde; 2011.

4. Menezes MAS, Garcia DC, Melo EV, Cipolotti R. Recém-nascidos prematuros assistidos pelo Método Canguru: avaliação de uma coorte do nascimento aos seis meses. Rev Paul Pediatr. 2014; 32(2):1717.

5. Oliveira CS, Casagrande GA, Grecco LC, Golin MO. Profile of preterm newborn hospitalized in intensive care unit of high complexity hospital. ABCS Health Sci. 
2015; 40(1):28-32.

6. Contim D, Moreira NR, José de Souza D, Passo MTD, Ferreira MBG, Simões

ALA. Classificação dos cuidados de enfermagem para crianças hospitalizadas: subsídio para práticas seguras. Rev Enferm Atenção Saúde. 2014; 3(2):29-40. 7. Roso CC, Costenaro RGS, Rangel RF, Jacobi CS, Mistura C, Trivisiol da Silva, C et al.Vivências de mães sobre a hospitalização do filho prematuro. Rev Enferm UFSM. 2014; 4(1):47-54. 8. Souza MHN, Paz EPA, Farias SNP, Ghelman LG, Mattos CX, Barros RR. Integrality as a dimension of nursing practice in mother-baby welcoming. Esc Anna Nery. 2013; 17(4):677- 82.

9. Costa MCG, Arantes MQ, Brito MDC. Neonatal ICU under the mother's view.

Rev Eletr Enf [Internet]. 2010 [citado 2015 Jul 15]; 12(4):698704. Disponível em: :http://dx.doi.org/10.5216/ree.v12i4.7130. 10. Fontanella BJB, Luchesi BM, Saidel MGB, Ricas J, Turato ER, Melo DG. Sampling in qualitative research: a proposal for procedures to detect theoretical saturation. Cad Saúde Pública. 2011; 27(2):389-94.

11. Lefevre F, Lefevre AM. Pesquisa de Representação Social: um enfoque qualiquantitativo: a metodologia do Discurso do Sujeito Coletivo. Brasília: Liber Livro Editora; 2012.

12. Araujo BBM, Rodrigues BMRD. Experiencias y perspectivas maternas en la internación del niño prematuro en unidad de terapia intensiva neonatal. Rev Esc Enferm USP. 2010; 44(4):865-72.

13. Balbino FS, Yamanaka CI, Balieiro MMF, Mandetta MA. Parent's support group as a transforming experience for families at a neonatal unit. Esc Anna Nery. 2015; 19(2): 297-302.

14. Gallegos-martinez, J; Reyeshernandez, J; Scochi, CGS. The hospitalized preterm newborn: The significance of parents' participation in the Neonatal Unit. Rev Latino-Am Enfermagem. 2013; 21(6):1360-6.
15. Favaro MSF, Peres RS, Santos MA. Avaliação do impacto da prematuridade na saúde mental de puérperas. Psico-USF. 2012; 17 (3):457-65.

16. Antunes BS, Paula CC, Padoin SMM, Trogahn TC, Rodrigures AP, Tronco CS. Internação do recém-nascido na Unidade Neonatal: significado para mãe. Rev RENE. 2014; 15(5):796-803. 17. Fernandes NGV, Batoca Silva EM. Fathers' experiences during the hospitalization of the premature newborn in the Neonatal Intensive Care Unit. Rev Bras Enferm. 2015; 4(4):107-15.

18. Cruz ARM, Oliveira MMC, Cardoso MVLML, Lúcio IML. Sentimentos e expectativas da mãe com filho prematuro em ventilação mecânica. Rev Eletr Enf. [Internet]. 2010 [citado 2015 Jul] 15]; 12(1):133-9. Disponível em: http://www.fen.ufg.br/revista/v12/n1/v12n1a16 .htm. 12(1):133-9. Disponível em: http://www.fen.ufg.br/revista/v12/n1/v12n1a16 .htm.

RECEBIDO: $23 / 06 / 2016$

APROVADO: $22 / 05 / 2017$

PUBLICADO: 31/07/2017 